\title{
Improving resolution in single-scan 2D spectroscopy
}

\author{
Philippe Pelupessy ${ }^{\mathrm{a}, *}$, Luminita Duma ${ }^{\mathrm{a}}$, Geoffrey Bodenhausen ${ }^{\mathrm{a}, \mathrm{b}}$ \\ ${ }^{a}$ Ecole Normale Supérieure, Département de Chimie, associé au CNRS, 24 rue Lhomond, 75231 Paris Cedex 05, France \\ ${ }^{\mathrm{b}}$ Ecole Polytechnique Fédérale de Lausanne, Laboratoire de Résonance Magnétique Biomoléculaire, Batochime, CH-1015 Lausanne, Switzerland
}

\section{A R T I C L E I N F O}

\section{Article history:}

Received 26 May 2008

Revised 26 June 2008

Available online 9 July 2008

\section{Keywords:}

NMR

Ultrafast spectroscopy

Aliasing

Undersampling

Band-selective pulses

Bipolar pulsed field gradient pairs

\begin{abstract}
A B S T R A C T
New schemes are introduced that allow one to improve the resolution in the indirect dimension of singlescan 'ultrafast' two-dimensional NMR spectra. The methods combine undersampling with band-selective pulses to recover signals that lie outside the detection bandwidth. The efficiency is illustrated for homonuclear total correlation spectroscopy (TOCSY) of quinidine.
\end{abstract}

(C) 2008 Elsevier Inc. All rights reserved.

\section{Introduction}

Nuclear magnetic resonance (NMR) spectroscopy has become an essential tool for studying a broad variety of systems and phenomena. Applications range from the search of new drugs to the characterization of internal dynamics of molecules [1]. The advent of two-dimensional (2D) methods made a decisive impact on the evolution of various techniques and greatly contributed to broaden the scope of applications. Traditional twodimensional experiments are intrinsically time consuming, since many $t_{1}$ increments have to be acquired in order to obtain 2D spectra with adequate digital resolution in the indirect $\omega_{1}$ dimension [2,3], even when samples with sufficient concentration or suitably enhanced nuclear polarization are available. Frydman and co-workers [4,5] have introduced a scheme inspired by echo planar imaging (EPI) [6], enabling the acquisition of a complete 2D NMR spectrum in a single scan, i.e., in less than a second. Thus, the time required to acquire multi-dimensional NMR experiments can be reduced by orders of magnitude. This advantage can be achieved by replacing the usual incrementation of the $t_{1}$-evolution interval by a spatial encoding of the phases across the sample, while decoding is performed with the help of alternating gradients applied during the detection period. The spatial encoding can be greatly improved by using adiabatic frequency-modulated pulses [7-9]. This new technique has been successfully combined with liquid chromatography [10] and with methods to increase the polarization of nuclear spins

\footnotetext{
* Corresponding author. Fax: +33144323397.

E-mail address: philippe.pelupessy@ens.fr (P. Pelupessy).
}

[11]. In addition, the method has been adapted for single-scan magnetic resonance imaging [12,13]. The detection element has been modified to suppress chemical shift evolution in order to obtain J-modulated spectra $[14,15]$.

In contrast to traditional approaches to 2D spectroscopy, the spectral resolution in single-scan experiments is not only limited by the inhomogeneity of the static field and by the homogeneous linewidths that are determined by relaxation, but also by the ability of the decoding gradient to 'unwind' the full spectrum in the indirect dimension. As a result, the spectral widths in the two dimensions are no longer independent of each other: the greater the spectral width that must be covered in either $\omega_{1}$ or $\omega_{2}$ dimensions, the poorer the digital resolution in the indirect $\omega_{1}$ dimension. The present study addresses these limitations by introducing new methods to improve the resolution in the indirect dimension of single-scan multi-dimensional spectra.

\section{Theory}

We shall briefly sum up the main characteristics of the basic single-scan 2D experiment sketched in Fig. 1 [7]. Effects of relaxation and of inhomogeneous fields will be neglected. The sequence starts with the excitation of spin $S$ followed by frequency encoding using two consecutive adiabatic pulses each of duration $\tau_{E}$ with a linear sweep of the radio-frequency (RF) carrier over a range $\Delta v_{a d}$. These two adiabatic encoding pulses are applied in the presence of two pulsed field gradients (PFGs) with the same strength $\boldsymbol{g}_{E}(\boldsymbol{r})$ but with opposite signs, in the manner of 'bipolar pulse pairs'. The phase $\phi_{E}$ of the coherence of a spin $S$ after frequency encoding depends on the position $\boldsymbol{r}$ of spin $S$ in the sample: 


\section{Excitation}

Evolution

Mixing Detection
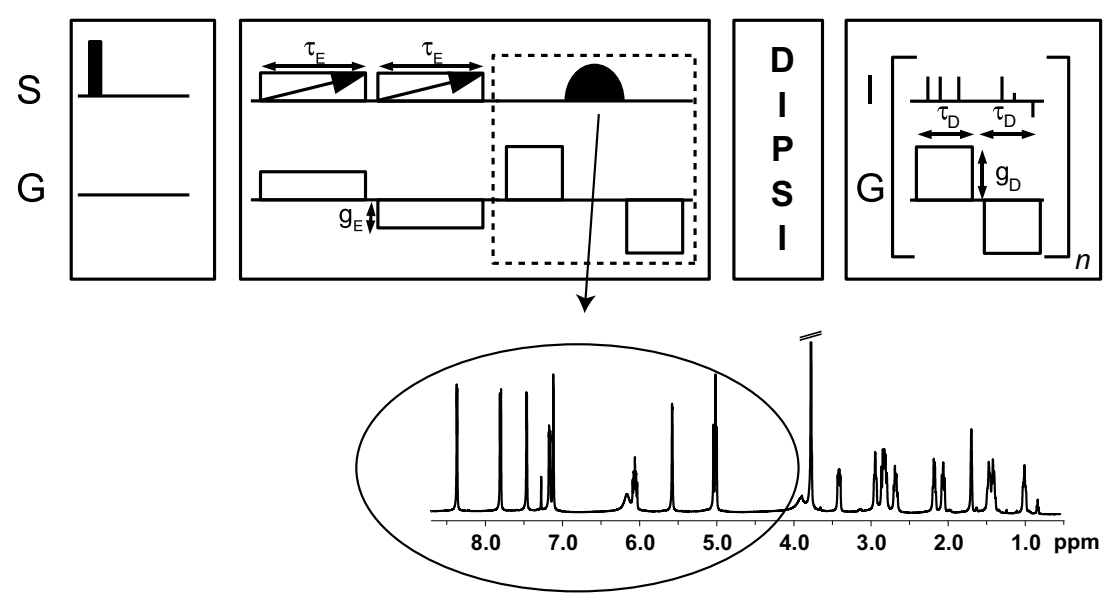

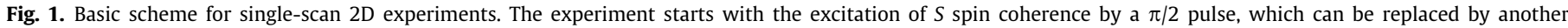

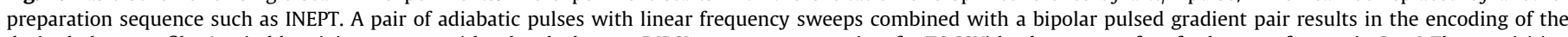

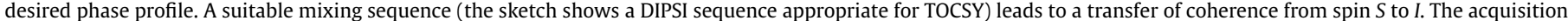

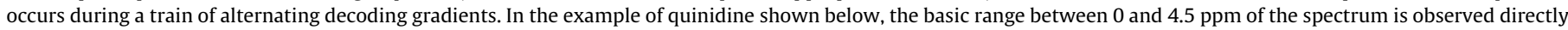

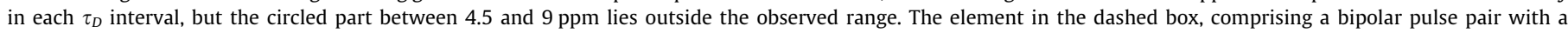

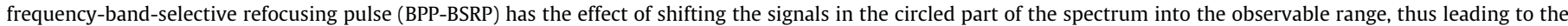

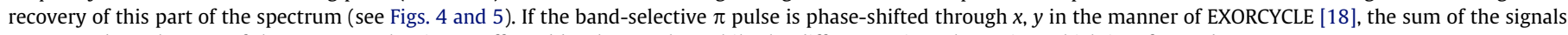
corresponds to the part of the spectrum that is not affected by the $\pi$ pulse, while the difference gives the region which is refocused.

$\phi_{E}=-\left\{4 \tau_{E} /\left(2 \pi \Delta v_{a d}\right)\right\} \Omega_{S} \gamma_{S} \boldsymbol{g}_{E}(\boldsymbol{r}) \cdot \boldsymbol{r}$

where $\gamma_{S}$ is the gyro-magnetic ratio of spin $S$, and $\Omega_{S}$ its chemical shift with respect to the RF carrier in the center of the sweeps. We shall henceforth assume that the gradient is applied along the $z$-axis, and hence replace $\boldsymbol{r}$ by $z$ and $\boldsymbol{g}_{E}$ by $g_{E}$. After the encoding period, the coherence can be transferred from spin $S$ to spin $I$ during a suitable mixing interval. The example of Fig. 1 shows a DIPSI (Decoupling In the Presence of Scalar Interactions) [16] sequence for total correlation spectroscopy (TOCSY) [17]. If we define $t_{1}$ to be the time running from the beginning of each positive lobe of the alternating decoding gradients, $\tau_{D}$ as the duration of each decoding gradient, and $n$ as the counter of the decoding gradient pair (each pair having a duration $2 \tau_{D}$ ), we can define a running variable $t_{2}=2 n \tau_{D}$, so that the signal acquired during the positive lobes of the alternating gradients of amplitude $g_{D}$ is given by

$s\left(z, t_{1}, n\right)=N P_{S, I} \exp \left[\mathrm{i} \phi_{E}+\mathrm{i} \gamma_{I} g_{D} z t_{1}+i \Omega_{I}\left(t_{1}+t_{2}\right)\right]$

where $N$ is a normalization factor, and $P_{S, I}$ is the probability that coherence transfer occurs from spin $S$ to $I$. If this transfer is associated with a phase-shift, $P_{I, S}$ may be a complex number. When normalizing the initial amplitude to one and integrating over the effective sample height $h$, one obtains the total signal:

$s\left(t_{1}, t_{2}\right)=P_{S, I} \operatorname{sinc}\left(-\alpha \Omega_{S}+\beta t_{1}\right) \exp \left[\mathrm{i} \Omega_{I}\left(t_{1}+t_{2}\right)\right]$

where $\alpha=\tau_{E} \gamma_{S} g_{E} h /\left(\pi \Delta v_{a d}\right)$ and $\beta=\gamma_{I} g_{D} h / 2$. The signals acquired during the negative lobes of the detection gradient pairs are mirror images of the ones recorded during the positive lobes, apart from a phase factor which depends on $\Omega_{I}$.

In traditional two-dimensional experiments, the digital resolution, i.e., the separation $\Delta v_{i}$ between neighboring points in the digitized spectra in the indirect $v_{1}=\omega_{1} /(2 \pi)$ dimension $(i=1)$ and in the direct $v_{2}=\omega_{2} /(2 \pi)$ dimension $(i=2)$ is determined by the corresponding acquisition times:

$\Delta v_{i}=1 /\left(N_{i} \Delta \mathrm{t}_{i}\right)=1 / t_{i}^{\max }$

where $N_{i}$ is the number of acquisition points, $\Delta t_{i}$ the increment, and $t_{i}^{\max }$ the acquisition time. For a given spectral width, which is inversely proportional to $\Delta t_{i}$, the digital resolution can always be im- proved by increasing the number $N_{i}$ of observed data points. Thus, the digital resolution parameters $\Delta v_{1}$ and $\Delta v_{2}$ in the two dimensions of traditional 2D spectra are independent of each other. An improved resolution in the indirect dimension requires a longer experimental time.

This tenet no longer holds in single-scan experiments. In the direct $\omega_{2}$ dimension, Eq. (4) is still valid, but there is no equivalent in the indirect $\omega_{1}$ dimension of single-scan experiments, since no Fourier transformation is performed. Therefore, to compare experiments, we have to use another definition of the resolution. In a traditional 2D experiment, the shape of a peak $s(v)$ that is centered at a frequency $v_{I}$ and results from the Fourier transformation of a truncated time-domain signal can be approximately described by:

$$
s_{i}(v)=\operatorname{sinc}\left[2 \pi\left(v-v_{I}\right) t_{i}^{\max }\right]
$$

We can define the spectral resolution as the interval between the maximum and the first zero passage of this sinc function:

$\Delta v_{i}=1 /\left(2 t_{i}^{\max }\right)$

For single-scan experiments, Eq. (3) describes a lineshape that is similar to Eq. (5) for conventional Fourier transform spectra. By analogy to Eq. (6), bearing in mind that the peaks appear at different points of time in the $\tau_{D}$ interval of single-scan experiments, we can define

$\Delta t_{1}=2 \pi / \gamma_{I} g_{D} h=1 / \Delta v_{D}^{I}$

where $\Delta t_{1}$ stands for the interval between the maximum and the first zero passage of Eq. (3), and $\Delta v_{D}^{I}$ is the frequency range that the decoding gradients $g_{D}$ impose on the $I$ spins. It is remarkable that the temporal resolution does not depend on the parameters of the initial encoding sequence. The lineshape in the indirect dimension is identical to that of a gradient echo. The linewidth is determined by the effective sample height $h$ and the strength $g_{D}$ of the decoding gradient, while the position of the echo depends on the chemical shift $\Omega_{S}$ of spin $S$. In units of frequency, the spectral resolution $\Delta v_{1}$ in the indirect domain is given by

$\Delta v_{1}=W_{1} \Delta t_{1} / \tau_{D}$ 
where the spectral width $W_{1}$ in the indirect dimension can be deduced from Eq. (3):

$W_{1}=\tau_{D} \beta / \alpha=\tau_{D} \gamma_{I} g_{D} \pi \Delta v_{a d} /\left(2 \tau_{E} \gamma_{S} g_{E}\right)$

The duration $\tau_{D}$ of each decoding gradient corresponds to half the dwell time $\Delta t_{2}$ in the direct dimension, hence:

$\Delta v_{1}=2 W_{1} /\left(\Delta t_{2} \Delta v_{D}^{I}\right)=2 W_{1} W_{2} / \Delta v_{D}^{I}$

The full width at half height, which is easy to determine experimentally, is related to $\Delta v_{1}$ as follows [15]:

$\Delta v_{1}^{F W H H}=1.21 \Delta v_{1}$

Thus, the spectral resolution $\Delta v_{1}$ in the indirect dimension depends on the strength of the decoding gradients and on the spectral widths in both indirect and direct dimensions. The greater the spectral width that needs to be covered in either dimension, the worse the spectral resolution in the indirect dimension. The development of strong pulsed field gradients with short switching delays and appropriately sturdy probes will therefore be crucial for improving the spectral quality in single-scan multi-dimensional experiments. However, very intense gradients may lead to signal losses because of translational diffusion $[19,20]$. The spectral resolution in the indirect dimension, when expressed in $\mathrm{ppm}$, is proportional to the strength of the static magnetic field. Thus, at higher $B_{0}$ fields, the resolution in the indirect dimension will be degraded. Note that an increase of the amplitude of the decoding gradients alone is not enough to improve the spectral resolution in the indirect dimension. Although the peaks will be sharper, they will be spread over a smaller time span because a larger spectral width $W_{1}$ is covered. If one covers the full spectral width with a given strength of the decoding gradient, increasing this gradient strength will merely add empty regions to the spectrum. In order to improve the resolution, the other parameters of Eq. (9) need to be adapted to restore the desired spectral width, $W_{1}$. For fixed spectral widths $W_{1}$ and $W_{2}$, the resolution in the indirect dimension improves with increasing decoding gradient strengths. Hereafter, we explore avenues to improve the resolution in the indirect dimension when the limits of the decoding gradients are attained.

In Fig. 2a, the poor spectral resolution is illustrated for a $500 \mathrm{mM}$ sample of quinidine in deuterated chloroform recorded at $295 \mathrm{~K}$. The experiment of Fig. 1 was carried out without mixing sequence (so that only signals along the diagonal of the twodimensional spectra are expected) in a static field $\mathrm{B}_{0}=14.1 \mathrm{~T}$ ( $600 \mathrm{MHz}$ for protons). The excitation element contains two consecutive adiabatic WURST (Wideband Uniform Rate and Smooth
Truncation) [21] pulses applied in the presence of two PFGs with the same strength but opposite signs. Both PFGs were applied along the $x$ and $z$ directions. The use of additional gradients along the $x$-axis does not change the resolution significantly but reduces the 'sinc wiggles' of the signals [22]. Each adiabatic encoding pulse had a duration $\tau_{E}=6 \mathrm{~ms}$ with a linear sweep of the RF carrier over a range $\Delta v_{a d}=40 \mathrm{kHz}$. In the direct dimension, a dwell-time $\Delta t_{2}=2 \tau_{D}=208 \mu \mathrm{s}$ is required to cover the full width of the proton spectrum of $4.8 \mathrm{kHz}$ width ( $8 \mathrm{ppm}$ ). This merely leaves $\tau_{D}=104 \mu \mathrm{s}$ for each lobe of the alternating decoding gradients in the acquisition interval. A total of $n=512$ gradient pairs with a sinusoidal smoothing of the initial and final $20 \mu$ s [23] amplitude profile of ca. $11 \mathrm{G} / \mathrm{cm}$, i.e., $20 \%$ of the maximum strength, have been used during signal acquisition which lasted about $106 \mathrm{~ms}$. A home-written program taking into account the smoothness of the amplitude profile, the number of gradient echoes and the duration of each decoding gradient was used to optimize the decoding pulse gradients. The resolution in the indirect dimension is inadequate for most practical purposes.

Since the maximum allowed strength $g_{D}$ of the decoding gradients is necessarily limited, the best way to increase the spectral resolution in the indirect dimension is to increase the duration $\tau_{D}$ of the decoding gradients. Unfortunately, this leads to a decrease of the spectral width in the direct $\omega_{2}$ dimension. In Fig. 2b and $c$ the dwell-time $\Delta t_{2}=2 \tau_{D}$ was first doubled and then quadrupled with respect to Fig. 2a. This corresponds to detection gradients of 208 and $416 \mu \mathrm{s}$, respectively (both with a sinusoidal smoothing of the initial and final $20 \mu \mathrm{s}$ ). Of course, the spectrum in the indirect $\omega_{1}$ dimension has to be "spread out" at the same time. This can be achieved either by prolonging the duration $\tau_{E}$ of the encoding gradients and concomitant adiabatic pulses, or by increasing the amplitudes of the encoding gradients. The latter solution is only possible if the sweep width of the adiabatic encoding pulses adequately covers the frequency range imposed on the $S$ spins by the gradients. If losses due to transverse relaxation, scalar couplings or diffusion during the encoding interval are significant, it is important to reduce the frequency range of the adiabatic pulse as much as possible $[7,15,24]$ and only a prolongation of the encoding gradients is feasible. Note that in our experiments the total length of the encoding period was only $12 \mathrm{~ms}$ so that the frequency range of the adiabatic pulse could safely be chosen large enough to absorb increases in the amplitude of the encoding gradient. The gain in resolution in the indirect $\omega_{1}$ dimension is evident. The peaks that lie outside the spectral width in the direct $\omega_{2}$ dimension are simply aliased in. For a sample height of $1.6 \mathrm{~cm}$, the theoretical a

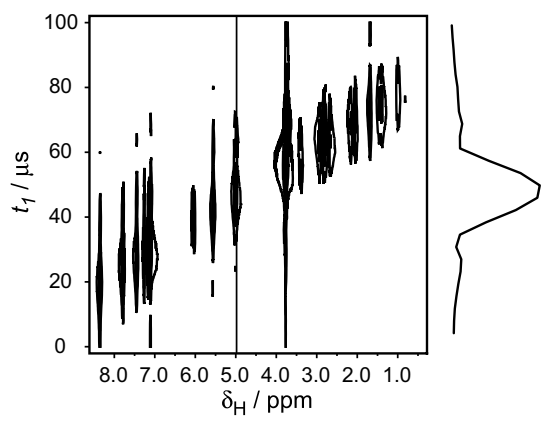

b

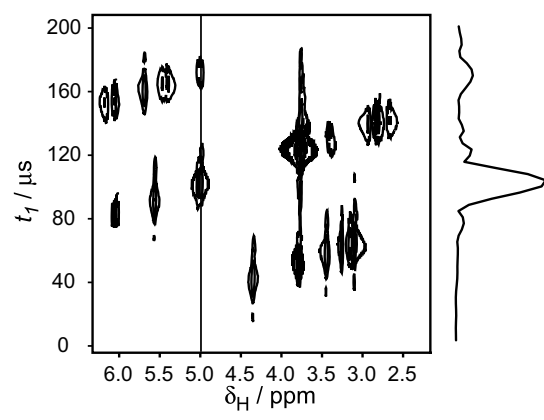

C

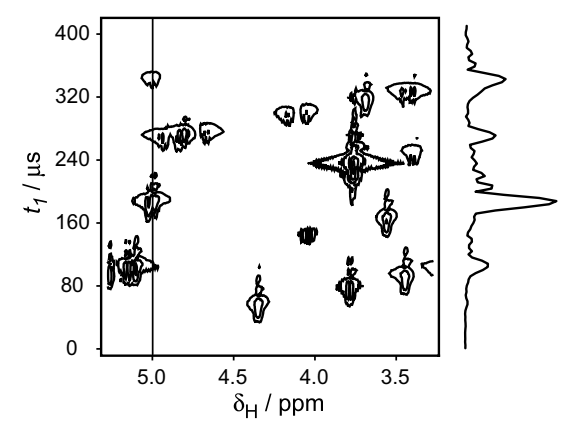

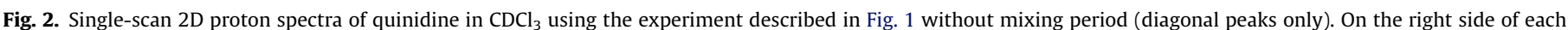

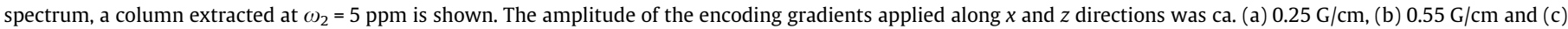

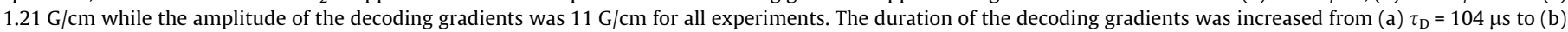

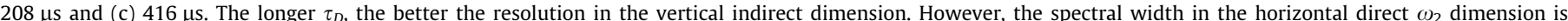

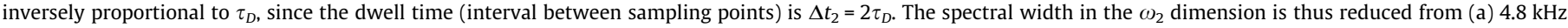
$(8.01 \mathrm{ppm})$ to (b) $2.4 \mathrm{kHz}(4.01 \mathrm{ppm})$ and (c) $1.2 \mathrm{kHz}(2.00 \mathrm{ppm})$. Consequently, peaks that lie outside the spectral width are aliased in the direct $\omega_{2}$ dimension. 
full width at half height of the signals in the indirect dimension, as can be deduced from Eqs. (10) and (11), is $16.1 \mu \mathrm{s}$. The widths at half height of the resonance at $5 \mathrm{ppm}$ in Fig. $2 \mathrm{a}-\mathrm{c}$ were $16.8 \pm 1.0,17 \pm 1.0$ and $17.2 \pm 1.0 \mu \mathrm{s}$, corresponding to $2.12,0.95$ and $0.43 \mathrm{ppm}$. The gains in resolution are better than would be expected from doubling or quadrupling the decoding gradient amplitudes. This can be explained by the fact that the transition period between the positive and negative decoding gradients (always maintained at $40 \mu \mathrm{s}$ ) takes percentage-wise less time when the duration of the decoding gradients increases.

The spectra in Fig. 3a and b were obtained with the same parameters as Fig. 2a and c, except that a DIPSI-2 mixing sequence of 22 ms duration was inserted (see Fig. 1) to bring about a transfer of coherence between all spins in the manner of TOCSY.

If the one-dimensional proton spectrum is known, the original positions of the peaks can easily be reconstructed. Powerful methods exist to choose a spectral width that is as small as possible without generating any ambiguities due to folding [25-27]. If the proton spectrum is unknown, one should repeat the experiment with a slightly different spectral width in the direct $\omega_{2}$ dimension. Peaks that are aliased move as shown in Fig. 3c. The price to pay for this method is a loss in sensitivity. The signal-to-noise ratio is inversely proportional to the square root of the dwell time $2 \tau_{D}$, since fewer points are acquired in the direct dimension if $2 \tau_{D}$ is increased. Like the signals, the noise is aliased into the reduced observable spectral width. Note that extending the duration of the decoding gradient while decreasing its amplitude does not reduce diffusion losses [15]. Hence, to obtain an optimum spectral resolution, one should always choose an amplitude that is as high as safely possible before increasing the duration.
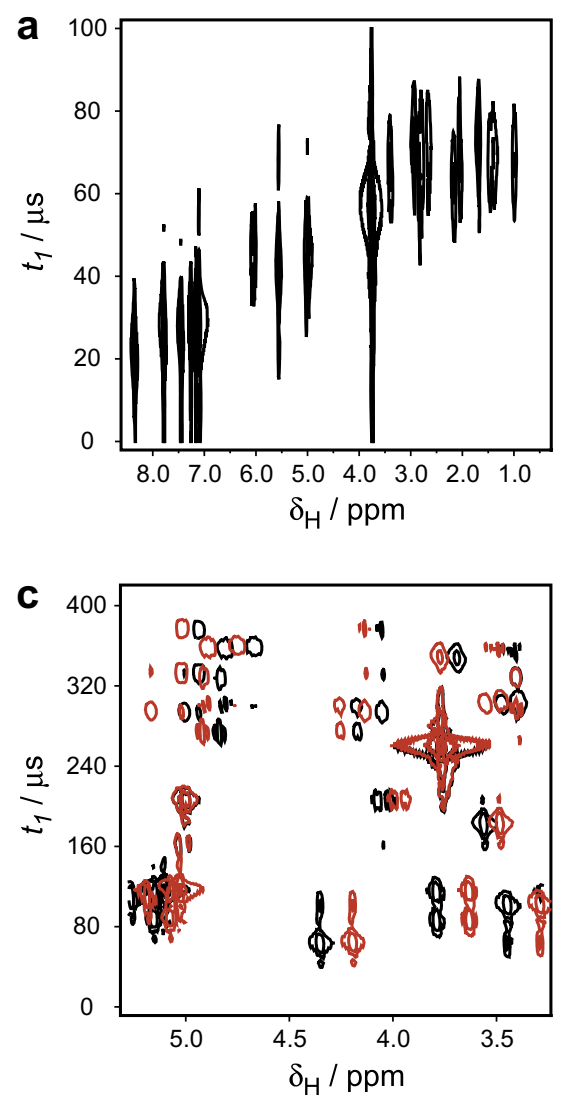

Another way to improve the resolution would be to compromise on the spectral width in the indirect $\omega_{1}$ dimension. According to Eq. (10), the smaller the spectral width $W_{1}$, the better the spectral resolution. In Fig. 4a, half of the spectrum of Fig. 2a has been "cut away" in order to increase the resolution. In order to recover the missing part of the spectrum, the experiment could be repeated after shifting the carrier frequency to the center of this part. A more efficient approach uses a band-selective refocusing pulse (BSRP) to recover the missing part of the spectrum. This $\pi$ pulse must be flanked by a bipolar pulse pair (BPP) comprising two gradients, as shown in the dashed box in Fig. 1. This gradient pair shifts the apparent frequencies of the spins that are refocused (circled resonances in Fig. 1) while it does not affect the region that is not touched by the pulse. By fine-tuning the parameters of the gradients, the missing part of the spectrum can be shifted at will. Fig. $4 \mathrm{~b}$ shows the effect of such a manipulation. Since a single $\pi$ pulse is applied, the peaks that are recovered appear along an "anti-diagonal". By shifting the phase of the band-selective pulse through $90^{\circ}$, in the manner of EXORCYCLE [18], the folded peaks change their sign. Thus, the sum of the spectra in Fig. 4c gives the original spectrum of Fig. 4a, while the difference in Fig. 4d gives the missing part. An advantage of this method is that it does not lead to a loss in signal-to-noise, apart from minor losses induced by relaxation during the bandselective pulse. If two separate experiments are carried out with a phase shift, the signal-to-noise increases with each scan, like in Hadamard spectroscopy [28]. This method is particularly advantageous when there are empty regions in the spectrum and could be generalized to shift more than one region by applying multiple BPP-BSRP elements.
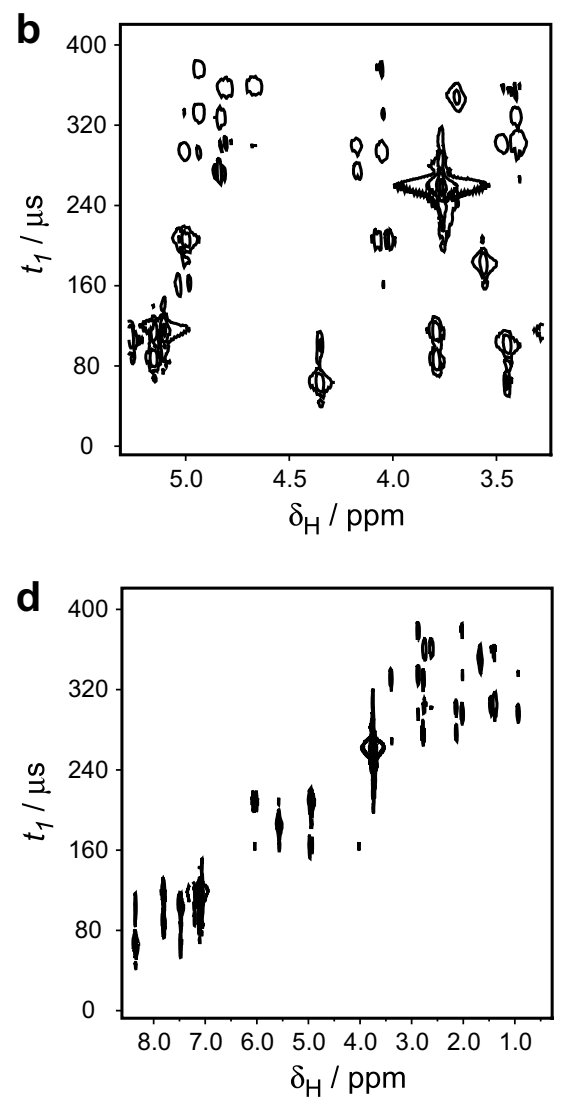

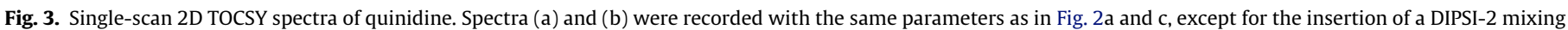

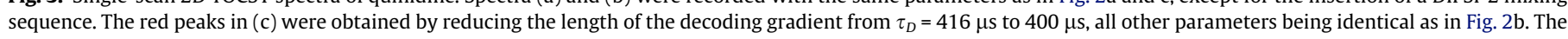

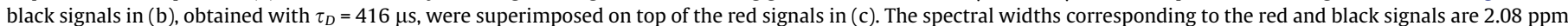

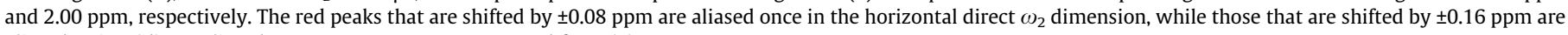
aliased twice. (d) Un-aliased TOCSY spectrum reconstructed from (c). 

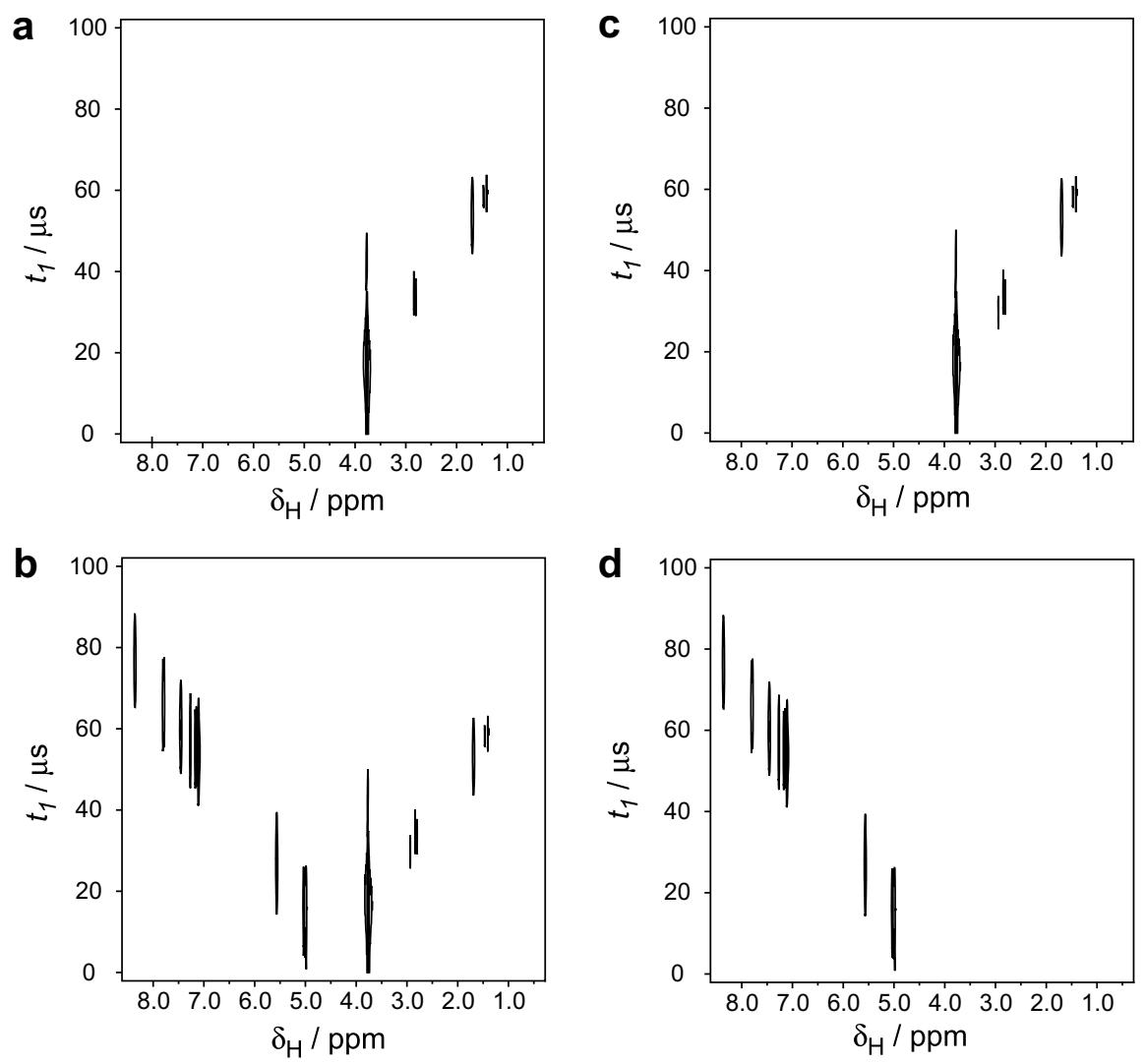

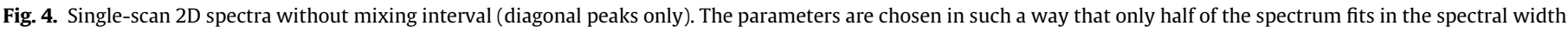

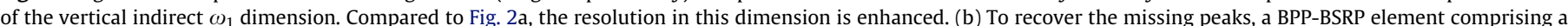

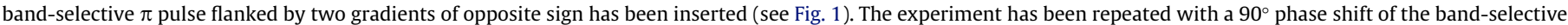
$\pi$ pulse (not shown). (c) The sum of the two experiments gives the initially selected region while (d) the difference results in the peaks that are missing in (a).
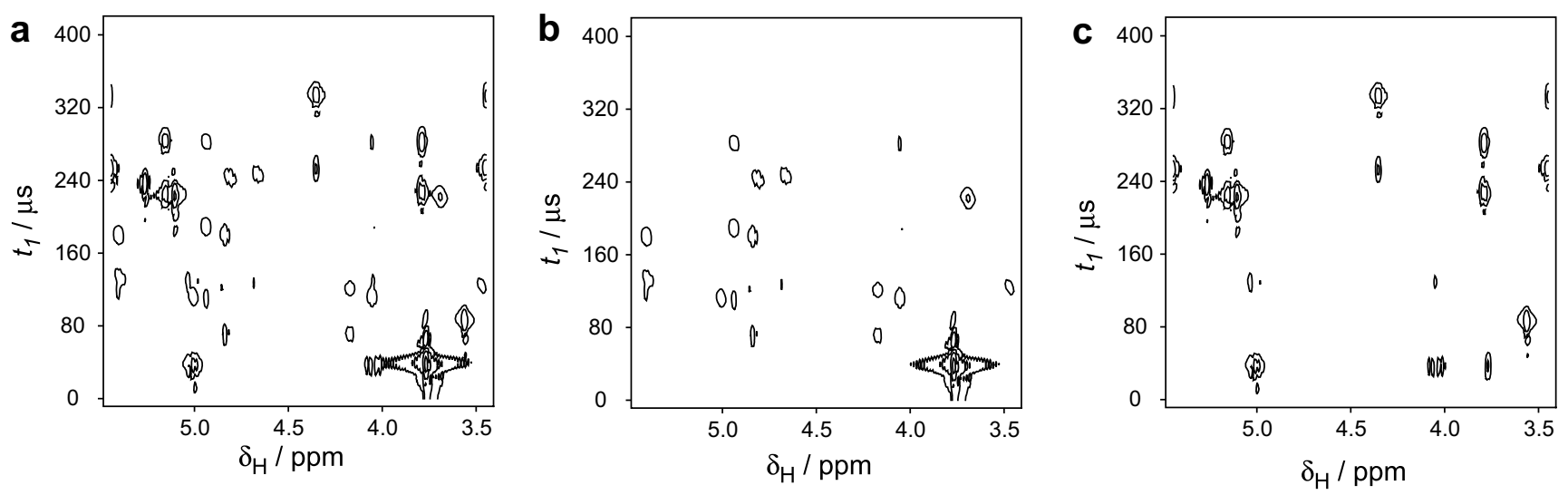

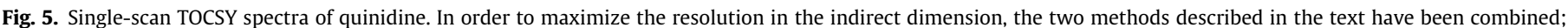

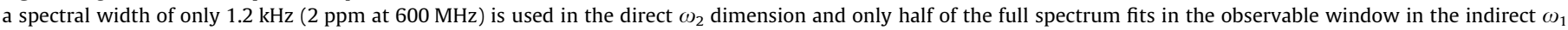
dimension, the remaining part being "recovered" by the band-selective pulses shown in Fig. 1. Fig. 5a-c are equivalent to Fig. 4b-d.

The two ways of improving the resolution presented in this work can be combined, i.e., the prolongation of the duration of the decoding gradients $\tau_{D}$, and the recovery of a missing part of the spectrum by a BPP-BSRP element. This is shown in Fig. $5 \mathrm{a}-\mathrm{c}$.

\section{Conclusions}

The spectral resolution in the indirect $\omega_{1}$ dimension of singlescan multi-dimensional experiments can be improved by increasing the strength of the alternating decoding gradients in the acquisition interval while keeping the same spectral width in both dimensions. In practice however, the gradient strength is limited for technical reasons and translational diffusion may cause signal losses if the gradients are too strong. Another approach is to limit the spectral width in the indirect dimension by using stronger and/ or longer encoding gradients. Band-selective refocusing pulses flanked by a bipolar pulse pair of gradients (a so-called BPP-BSRP element) can then recover peaks that lie outside the detectable spectral width in the indirect dimension. As an alternative, the dwell time in the direct $\omega_{2}$ dimension can be prolonged to make room for longer decoding gradients. This leads to a narrower spectral width in the direct dimension, and hence to aliasing, but no 
signals are lost. The two methods can be combined in order to further improve the digital resolution.

\section{Acknowledgments}

The authors are indebted to the Centre National de la Recherche Scientifique (CNRS, France), the Agence Nationale pour la Recherche (ANR, France), the Integrated Infrastructure Initiative (I3) of the 6th Framework Program of the EC (Contract \# RII3-026145, EU-NMR), the Fonds National de la Recherche Scientifique (FNRS, Switzerland), and the Commission pour la Technologie et l'Innovation (CTI, Switzerland).

\section{References}

[1] D.M. Grant, R.K. Harris (Eds.), Encyclopedia of Nuclear of Magnetic Resonance, John Wiley \& Sons Ltd., Chichester, 1996.

[2] J. Jeener, Lecture at International Ampère Summer School, Basko Polje, Yugoslavia, 1971

[3] W.P. Aue, E. Bartholdi, R.R. Ernst, 2-Dimensional spectroscopy-application to nuclear magnetic resonance, J. Chem. Phys. 64 (1976) 2229-2246.

[4] L. Frydman, T. Scherf, A. Lupulescu, The acquisition of multidimensional NMR spectra within a single scan, Proc. Natl. Acad. Sci. USA 99 (2002) 15858-15862.

[5] L. Frydman, A. Lupulescu, T. Scherf, Principles and features of single-scan twodimensional NMR spectroscopy, J. Am. Chem. Soc. 125 (2003) 9204-9217.

[6] M.K. Stehling, R. Turner, P. Mansfield, Echo-planar imaging-magneticresonance-imaging in a fraction of a second, Science 254 (1991) 43-50.

[7] P. Pelupessy, Adiabatic single scan two-dimensional NMR spectroscopy, J. Am. Chem. Soc. 125 (2003) 12345-12350.

[8] N.S. Andersen, W. Kockenberger, A simple approach for phase-modulated single-scan 2D NMR spectroscopy, Magn. Reson. Chem. 43 (2005) 795-797.

[9] A. Tal, B. Shapira, L. Frydman, A continuous phase-modulated approach to spatial encoding in ultrafast 2D NMR spectroscopy, J. Magn. Reson. 176 (2005) 107-114.

[10] B. Shapira, A. Karton, D. Aronzon, L. Frydman, Real-time 2D NMR identification of analytes undergoing continuous chromatographic separation, J. Am. Chem. Soc. 126 (2004) 1262-1265.

[11] L. Frydman, D. Blazina, Ultrafast two-dimensional nuclear magnetic resonance spectroscopy of hyperpolarized solutions, Nat. Phys. 3 (2007) 415-419.
[12] Y. Shrot, L. Frydman, Spatially encoded NMR and the acquisition of 2D magnetic resonance images within a single scan, J. Magn. Reson. 172 (2005) 179-190.

[13] A. Tal, L. Frydman, Spectroscopic imaging from spatially-encoded single-scan multidimensional MRI data, J. Magn. Reson. 189 (2007) 46-58.

[14] P. Giraudeau, S. Akoka, A new detection scheme for ultrafast 2D J-resolved spectroscopy, J. Magn. Reson. 186 (2007) 352-357.

[15] P. Giraudeau, S. Akoka, Resolution and sensitivity aspects of ultrafast Jresolved 2D NMR spectra, J. Magn. Reson. 190 (2008) 339-345.

[16] L. Braunschweiler, R.R. Ernst, Coherence transfer by isotropic mixingapplication to proton correlation spectroscopy, J. Magn. Reson. 53 (1983) 521-528.

[17] A.J. Shaka, C.J. Lee, A. Pines, Iterative schemes for bilinear operatorsapplication to spin decoupling, J. Magn. Reson. 77 (1988) 274-293.

[18] G. Bodenhausen, R. Freeman, D.L. Turner, Suppression of artifacts in 2dimensional J-spectroscopy, J. Magn. Reson. 27 (1977) 511-514.

[19] P. Giraudeau, S. Akoka, Sources of sensitivity losses in ultrafast 2D NMR, J Magn. Reson. 192 (2008) 151-158.

[20] Y. Shrot, L. Frydman, The effects of molecular diffusion in ultrafast two-dimensional nuclear magnetic resonance, J. Chem. Phys. 128 (2008) 15.

[21] E. Kupce, R. Freeman, Adiabatic pulses for wide-band inversion and broadband decoupling, J. Magn. Reson. Ser. A 115 (1995) 273-276.

[22] K.E. Cano, M.J. Thrippleton, J. Keeler, A.J. Shaka, Cascaded z-filters for efficient single-scan suppression of zero-quantum coherence, J. Magn. Reson. 167 (2004) 291-297.

[23] J.M. Böhlen, G. Bodenhausen, Experimental aspects of Chirp NMRspectroscopy, J. Magn. Reson. Ser. A 102 (1993) 293-301.

[24] B. Shapira, Y. Shrot, L. Frydman, Symmetric spatial encoding in ultrafast 2D NMR spectroscopy, J. Magn. Reson. 178 (2006) 33-41.

[25] D. Jeannerat, High resolution in heteronuclear H-1-C-13 NMR experiments by optimizing spectral aliasing with one-dimensional carbon data, Magn. Reson. Chem. 41 (2003) 3-17.

[26] D. Jeannerat, Computer optimized spectral aliasing in the indirect dimension of $\mathrm{H}-1-\mathrm{C}-13$ heteronuclear 2D NMR experiments. A new algorithm and examples of applications to small molecules, J. Magn. Reson. 186 (2007) 112122.

[27] E. Lescop, P. Schanda, R. Rasia, B. Brutscher, Automated spectral compression for fast multidimensional NMR and increased time resolution in real-time NMR spectroscopy, J. Am. Chem. Soc. 129 (2007) 2756-2757.

[28] E. Kupče, T. Nishida, R. Freeman, Hadamard NMR spectroscopy, Prog. NMR Spectrosc. 42 (2003) 95-122. 